\title{
Etude du mode de galerie dans un microdisque denté 2D avec une méthode semi-analytique à base de différences finies
}

\author{
Kien Phan Huy, David Amans, Alain Morand et Pierre Benech
}

\author{
IMEP, INPG-UJF-CNRS, 23 avenue des martyrs BP 257, 38016 Grenoble Cedex 1, France.
}

\begin{abstract}
Résumé - Un modèle décrivant le mode de galerie (MG) dans un microdisque denté a été développé. Basé sur un formalisme de Floquet-Bloch, le modèle décrit le champ de manière analytique à l'intérieur et à l'extérieur du disque et de manière numérique au niveau des dents. Le modèle calcule la longueur d'onde de résonance et le coefficient de qualité du MG. Confronté à la FDTD, il montre d'excellents résultats, tout en étant beaucoup plus rapide (quelques seconde pour quelques heurs en FDTD). D'autres microstructurations sur la périphérie du disque sont présentées et simulées avec ce type de méthode. Elle permet notamment d'optimiser des anneaux pour être dans un régime quasimonomode.
\end{abstract}

Mots-clefs - Microgear, résonateur diélectrique, mode de galerie.

\section{INTRODUCTION}

Les microdisques fabriqués à l'aide des technologies de la microélectronique ( $\mathrm{Si}, \mathrm{InP}, \mathrm{GaAs}$..) font aujourd'hui l'objet de plusieurs études et de programmes de développement. En effet, les modes de galerie se propageant dans ces disques offrent des coefficients de qualités spectaculaires permettant la réalisation de lasers doués de très bas seuils. Cependant leur multi-modicité pose un vrai problème, c'est pourquoi $\mathrm{T}$. baba $^{1}$ ont proposé la structure du microdisque denté qui est un microdisque entouré d'un réseau de Bragg circulaire comme le montre la figure (1). Les modes ne respectant pas la condition de Bragg sont alors diffractés vers l'extérieur de la cavité, réduisant considérablement le coefficient de qualité des modes parasites (comme le mode impair). Par contre le mode pair, vérifiant la condition de Bragg, voit son coefficient de qualité augmenter. Les microdisques dentés ont fait l'objet d'études numériques à travers des calculs FDTD et des méthodes de calculs intégrales ${ }^{2}$.

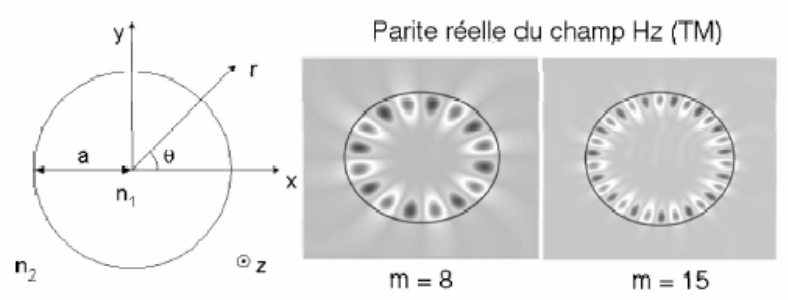

(a)

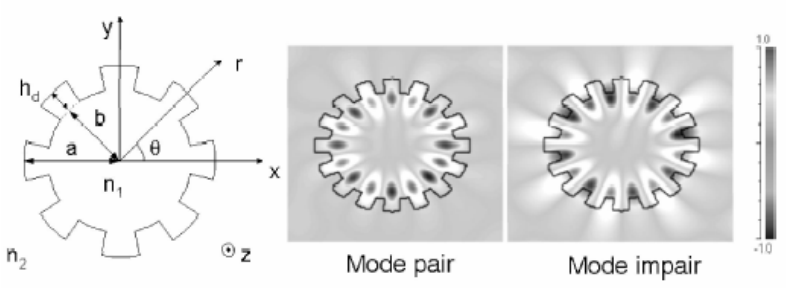

(b)

Fig. 1. (a) Structure du disque 2D sans déformation entouré d'air $\left(\mathrm{n}_{1}=2.2\right)$. Deux modes de galerie d'ordre azimutal $\mathrm{m}=8$ et $m=15$. (b) Structure du disque denté $2 \mathrm{D}$ entouré d'air $\left(\mathrm{n}_{1}=\right.$ 2.2). Deux modes de galerie pair et impair d'ordre azimutal $\mathrm{m}=8$.

Dans cet article, nous présentons une méthode semianalytique basée sur la théorie de Floquet-Bloch. Elle reprend la description analytiques des modes propres du disque en introduisant une résolution numérique de manière locale au niveau du réseau de Bragg. Cette méthode a l'avantage d'être harmonique et modale, ce qui la rend supérieur aux modèles de perturbation qui peuvent difficilement modéliser la déformation des champs. Comparée à la FDTD, elle montre de très bons résultats tout en étant plus rapide et sans souffrir des rugosités dues à un maillage cartésien. On se place dans le cas d'un problème $2 \mathrm{D}$ (cylindre) dans un système à coordonnées cylindriques $(\mathrm{r}, \theta, \mathrm{z})$ comme sur la figure 1 . considérons le microdisque denté de rayon extérieur a et de rayon intérieur $b=a-h_{d}$ d'indice de réfraction $n_{1}$ et entouré de $\mathrm{n}_{2}$. La résolution des équations de Maxwell se sépare en trois parties. Tout d'abord, on traite le problème analytiquement pour $\mathrm{r}<\mathrm{b}$ et $\mathrm{r}>\mathrm{a}$, car il est similaire au disque simple. Puis on résout les équations de Maxwell dans les dents selon un formalisme de différences finies. Enfin, on raccorde ces solutions pour assurer les conditions de continuité.

\section{RESOLUTION ANALYTIQUE DANS UN DISQUE SIMPLE}

Considérons le disque simple de la figure (1). On sait que les solutions des équations de Maxwell vont se scinder en deux groupes TE $\left(\mathrm{E}_{\mathrm{z}}, \mathrm{H}_{\mathrm{r}}, \mathrm{H}_{\theta}\right)$ et $\mathrm{TM}\left(\mathrm{H}_{\mathrm{z}}, \mathrm{E}_{\mathrm{r}}\right.$, $\left.\mathrm{E}_{\theta}\right)$. comme on s'intéresse aux modes de galerie qui se propagent selon $\theta$, on peut déjà imposer la forme des solutions : 


$$
\Psi_{m}=\Psi_{m}(r) \cos (m \theta) e^{j \omega t}
$$

Où $\mathrm{m}$ est l'ordre azimutal ou constante de propagation selon $\theta, \psi_{\mathrm{m}}$ est la composante $\mathrm{z}$ du champ électrique en polarisation TM, du champ magnétique en TE et $\omega$ la pulsation. Le cosinus est pris pour décrire le mode pair (sinus pour le mode impair). En reportant cette expression dans 1 'équation d'Helmoltz en coordonnées cylindriques pour la composante $\mathrm{z}$ du champ, on obtient :

$$
r^{2} \frac{\partial^{2} \Psi_{m}}{\partial r^{2}}+r \frac{\partial \Psi_{m}}{\partial r}+\left[\frac{\omega}{c} n_{1}^{2} r^{2}-m^{2}\right] \Psi_{m}=0
$$

où $\mathrm{c}$ est la vitesse de la lumière. Les solutions de cette équation sont des fonctions Bessels. Pour $\mathrm{r}<\mathrm{b}$, le champ est décrit par une onde stationnaire, la fonction de Bessel d'ordre $\mathrm{m} \mathrm{J}_{\mathrm{m}}\left(\omega \mathrm{n}_{1} \mathrm{r} / \mathrm{c}\right)$. Pour $\mathrm{r}>\mathrm{a}$, l'onde stationnaire se decompose en deux ondes propagatives et contrapropagatives, les fonctions de Hankel de première $\mathrm{H}_{\mathrm{m}}^{1}\left(\omega_{2} \mathrm{r} / \mathrm{c}\right)$ et seconde espèce $\mathrm{H}_{\mathrm{m}}^{2}\left(\omega_{2} \mathrm{r} / \mathrm{c}\right)$ dont les poids respectifs seront déterminés par les conditions de continuités.

\section{RESOLUTION NUMERIQUE DANS LE RESEAU DE BRAGG}

Le réseau de Bragg peut être décrit comme une decomposition en série de Fourier. A l'intérieur des dents, la permittivité diélectrique s'écrit $\varepsilon_{\mathrm{r}}(\theta)=$ $\sum b_{k} \cos (k m \theta)$ où $b_{k}$ est le poids de l'harmonique de pulsation $\mathrm{km}$ avec $\mathrm{k}$ allant de 0 à l'infini. Pour la polarisation TE les équations de Maxwell donnent les équations suivantes où $\mu_{0}$ est la perméabilité magnétique et $\varepsilon_{0}$ est la permittivité électrique du vide.

$$
\begin{array}{rlc}
-j \omega \mu_{0} H_{z} & = & \frac{1}{r}\left[\frac{\partial r E_{\theta}}{\partial r}-\frac{\partial E_{r}}{\partial \theta}\right] \\
j \omega \epsilon_{0} \epsilon_{r} E_{r} & = & \frac{1}{r} \frac{\partial H_{z}}{\partial \theta} \\
j \omega \epsilon_{0} \epsilon_{r} E_{\theta} & = & -\frac{\partial H_{z}}{\partial r}
\end{array}
$$

Le réseau de Bragg déforme le champ. Dès lors, on ne peut plus faire l'hypothèse précédente. Cela indique que la variation azimutal du mode ne sera plus une harmonique pure $\mathrm{m}$ mais une somme d'harmoniques $(\mathrm{nm})$ avec $\mathrm{n}$ entier comme sur les équations suivantes :

$$
\begin{aligned}
& H_{z}=\sum_{n} f_{n}(r) \cos (n m \theta) \\
& E_{r}=\sum_{n} h_{n}(r) \sin (n m \theta) \\
& E_{0}=\sum_{n} g_{n}(r) \cos (n m \theta)
\end{aligned}
$$

Après projections sur chaque harmonique $\mathrm{nm}$, on a trois $\mathrm{n}$ inconnus pour trois $\mathrm{n}$ équations. En remplaçant, les champs dans l'équation (3) par leurs expressions (4). On peut exprimer la matrice de passage $\left(\mathrm{C}_{\mathrm{p}, \mathrm{n}}\right)$ avec $\mathrm{p}$ et $\mathrm{n}$ entiers entre $h_{p}$ et $f_{n}$. On peut alors obtenir le système suivant :

$$
\begin{aligned}
\frac{\partial r g_{p}(r)}{\partial r}= & -j \omega \mu_{0} r f_{p}(r)+p m \sum_{n} c_{p, n} f_{n}(r) \\
\frac{\partial f_{p}(r)}{\partial r}= & -j \frac{1}{2} \omega \epsilon_{0} b_{0} g_{p}(r) \\
& -j \omega \epsilon_{0} \sum_{n} \frac{1}{2}\left[b_{p+n}+b_{|p-n|}\right] g_{n}(r) .
\end{aligned}
$$

On discrétise alors le problème en $\mathrm{r}$ et on résout le système numériquement à partir des conditions aux limites en b, obtenues grâce au calcul du champ sous le réseau fait au paragraphe précédent.

\section{CONDITIONS DE CONTINUITES}

Les solutions à raccorder sont récapitulés ci-dessous :

$$
\mathrm{r}<\mathrm{b}
$$

$$
\begin{array}{ccc}
H_{z} & : & \sum_{n} C_{n} J_{n m} \cos (n m \theta) \\
E_{\theta} & : & -\frac{k_{0} n_{1}}{j \omega \epsilon \epsilon_{r}} \sum_{n} C_{n} J_{n m}^{\prime} \cos (n m \theta)
\end{array}
$$

$$
\begin{aligned}
& \frac{\mathrm{b}<\mathrm{r}<\mathrm{a}}{\sum_{n} f_{n} \cos (n m \theta)} \\
& \frac{\sum_{n} g_{n} \cos (n m \theta)}{\mathrm{r}>\mathrm{a}} \\
& \frac{\sum_{n}\left[A_{n} H_{n m}^{1}+B_{n} H_{n m}^{2}\right] \cos (n m \theta)}{-\frac{k_{0} n_{0}}{j \omega \epsilon \epsilon_{r}} \sum_{n}\left[A_{n} H_{n m}^{\prime 1}+B_{n} H_{n m}^{\prime 2}\right] \cos (n m \theta)}
\end{aligned}
$$

On a bien deux $\mathrm{n}$ équations et trois $\mathrm{n}$ paramètres. En oscillation forcée, on peut donc imposer une excitation en fixant les $A_{n}$ qui sont les coefficients des ondes $\mathrm{H}^{1}{ }_{n m}$ entrant dans le disque et en déduire les poids $C_{n}$ des ondes $\mathrm{J}_{\mathrm{nm}}$ transmises à l'intérieur du disque et les poids $\mathrm{B}_{\mathrm{n}}$ des ondes réfléchies $\mathrm{H}^{2}{ }_{\mathrm{nm}}$. En oscillation libre, on fixe les $A_{n}$ à zéro, on se place donc un fonctionnement du type laser (sans source extérieur). Le système d'équations a alors des solutions si et seulement si le déterminant du système est nul. A l'aide d'un algorithme de newtonraphson on peut déterminer les pulsations complexes de résonance qui annule le déterminant. $\mathrm{Si}$ on considère la plus petite $\omega_{c}$ en valeur absolue, elle correspond au mode de galerie, on a alors: $\omega_{\text {res }}=\left|\omega_{\mathrm{c}}\right|$ et $\mathrm{Q}=\left|\omega_{\mathrm{c}}\right| /\left(2 \operatorname{Imag}\left(\omega_{\mathrm{c}}\right)\right)$.

\section{RESULTATS}

Nos résultats ont été comparés avec ceux obtenus avec une FDTD. Comme le montre la figure 2, nous obtenons un très bon accord avec les résultats numériques tout en 
étant beaucoup plus rapide. En effet, un point de la courbe FDTD représente plus d'une heure et demi de simulation contre trente six secondes pour les trente points de la courbe avec notre modèle. Le mode pair a la particularité d'avoir un coefficient de qualité qui atteint une valeur maximum pour une profondeur de trous donnée. Au contraire, le coefficient de qualité du mode impair décroît avec la profondeur des trous. Cette sélectivité peut permettre de diminuer le seuil laser si on utilise ce type de structure. On montre aussi que le maillage cartésien de la FDTD provoque des pertes par diffraction qui diminuent le coefficient de qualité du résonateur.
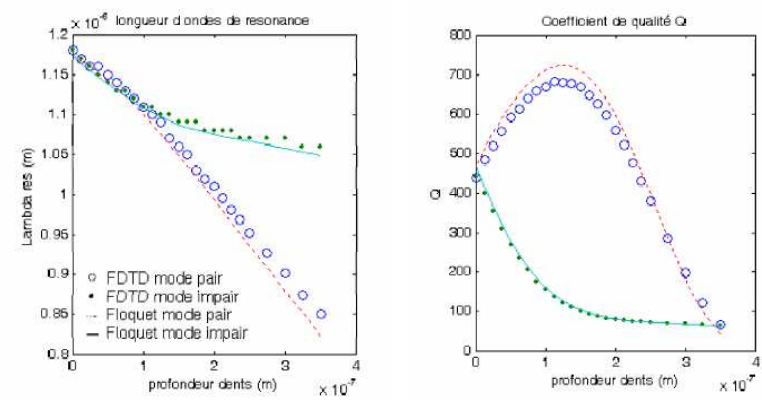

Fig. 2. Comparaison entre notre modèle et la simulation FDTD ( $a=1 \mu \mathrm{m}, \mathrm{n}_{1}=2.2$ et $\left.\mathrm{n}_{2}=0\right)$. La longueur d'onde et le coefficient de qualité du mode $\mathrm{m}=8$ en polarisation TE sont calculés en fonction de la profondeur des dents
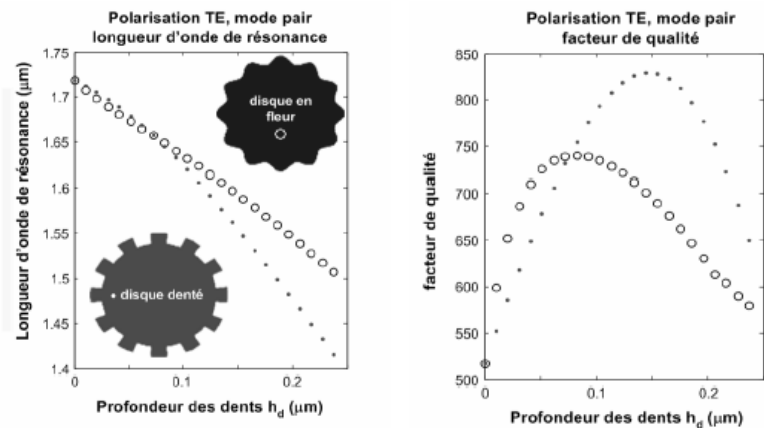

Fig. 3. Comparaison en terme de facteur de qualité d'un disque denté et d'un disque en fleur $\left(\mathrm{a}=1 \mu \mathrm{m}, \mathrm{n}_{1}=2.63\right.$ et $\mathrm{n}_{2}=1$ ).

Une autre micro-structuration en périphérie du disque a été étudiée. Le profil azimutal carré de $\varepsilon_{\mathrm{r}}$ est remplacé par un profil sinusoïdal. Sur la figure (3), les évolutions de la longueur d'onde de résonance et du facteur de qualité pour le micro-disque denté et le disque en fleur sont présentées en fonction de la profondeur des dents. Pour deux structures de rayon et d'indice de réfraction équivalents, la structure optimale du disque denté se révèle plus efficace que celle du résonateur en fleur. Le profil sinusoïdal de la fleur implique un rapport cyclique variable de $\varepsilon_{\mathrm{r}}$ selon $\mathrm{r}$. Le rapport cyclique n'étant pas le même à la base et à la pointe des dents, il en résulte un couplage plus fort sur les harmoniques d'ordre élevé $(\mathrm{km}>2 \mathrm{~m})$ lorsque le rapport cyclique s'éloigne de $50 \%$. Cela induit une diminution du facteur de qualité. Il est donc primordial d'optimiser la technologie pour avoir les meilleurs rapports de forme possible au niveau des gravures.

Le disque denté permet d'amoindrir considérablement les modes d'ordre azimutal parasites. Néanmoins, il existe pour un même ordre azimutal de nombreux modes d'ordre radial différents. Pour obtenir une structure quasi monomode il faut donc supprimer les modes d'ordre radial parasites. C'est pourquoi nous présentons ici un anneau denté. La majeure partie des démonstrations expérimentales d'anneaux montrent des anneaux non optimisés. En effet, les anneaux sont souvent considérés à tort comme des guides monomodes courbes. Or il est assez facile de montrer qu'un anneau dont la largeur est celle d'un guide droit monomode possède un facteur de qualité inférieur au disque de rayon équivalent. La figure (4) montre ainsi qu'un anneau d'InP de la largeur d'un guide droit monomode (généralement de l'ordre de $0,3 \mu \mathrm{m}$ ) possède un facteur de qualité de l'ordre de 100 contre 500 pour le disque plein. La largeur de l'anneau n'est donc pas un paramètre anodin.

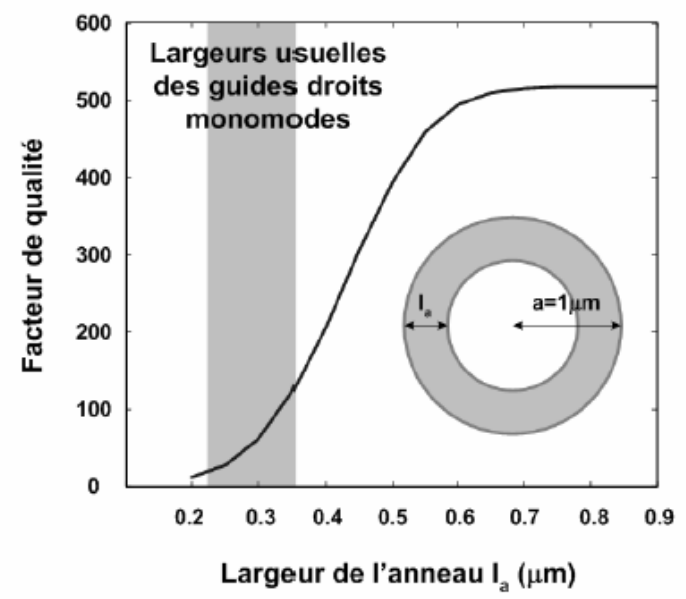

Fig. 4. Calcul du facteur de qualité d'un anneau d'InP $(n=2$. 63 ) entouré d'air en fonction de la largeur de l'anneau à rayon extérieur constant $\mathrm{a}=1 \mu \mathrm{m}$.

Sur la figure (5), nous avons représenté l'évolution du facteur de qualité en fonction du rayon intérieur de l'anneau $\mathrm{R}_{0}$ et de la profondeur des dents $\mathrm{h}_{\mathrm{d}}$ d'un anneau de rayon extérieur $1.5 \mu \mathrm{m}$. Nous montrons les résultats pour un ordre azimutal $\mathrm{m}=10$ et deux ordres radiaux $1=$ 0 et $1=1$. Nous remarquons que les deux modes possèdent une structure optimale différente. Ainsi, si on considère la structure indiquée par le cercle vert, nous aurons un mode de galerie $(\mathrm{m}=10,1=0)$ fortement favorisé et un mode $(\mathrm{m}=10,1=1)$ très défavorisé. Nous avons une structure quasi monomode. Notons que l'anneau a alors une largeur bien supérieure à celle d'un guide droit monomode. 
(a) Facteur de qualité du mode de galerie $m=10,1=0$

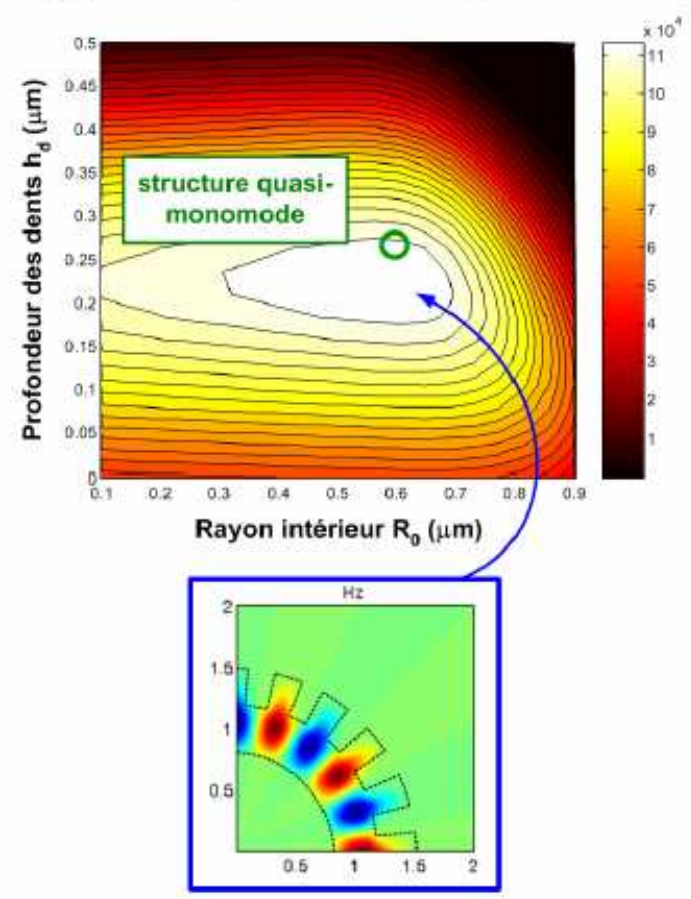

(b) Facteur de qualité du mode de galerie $m=10, \mathrm{l}=1$

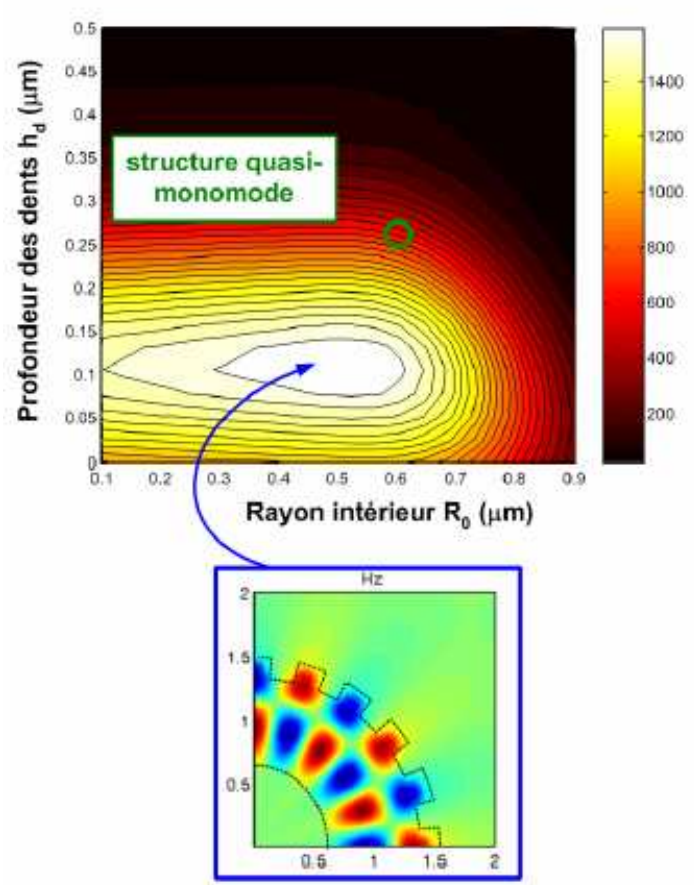

Fig. 5. La structure étudiée est un anneau de rayon intérieur $\mathrm{R}_{0}$, de rayon extérieur $\mathrm{a}=1,5 \mu \mathrm{m}$ au bord duquel un réseau de Bragg est inscrit. La profondeur des dents est notée $h_{d}$ avec $n_{2}$ $=2.63$ et $n_{1}=1$. (a) Nous représentons la variation du facteur de qualité du mode de galerie $\mathrm{TE}(\mathrm{m}=10,1=0)$ en fonction de $\mathrm{R}_{0}$ et $\mathrm{h}_{\mathrm{d}}$. La carte de champ correspondant à l'optimisation pour ce mode est montrée. (b) On montre la variation du facteur de qualité pour le mode $\mathrm{TE}(\mathrm{m}=10,1=1)$. L'optimum n'est pas le même.

\section{CONCLUSION}

Nous avons présenté un modèle semi-analytique pour simuler les micro-structurations d'un disque. Comparé à la FDTD, ce modèle de Floquet-Bloch s'est montré particulièrement rapide, tout en étant précis et stable. Nous avons pu ainsi étudier la structure du disque denté et montrer les propriétés de sélectivité modale de ce composant. Puis, nous avons abordé les effets des processus technologiques sur ce composant en étudiant le disque en fleur. Nous avons alors présenté le composant quasi-monomode optimum qu'est l'anneau denté.

\section{REFERENCES}

[1] M. Fujita and T. Baba, «Microgear laser», Apllied Physics Letters, vol. 80, pp. 2051-2053, March 2002.

[2] S.V. Boriskina, T.M Benson, P. Sewell and A.I. Nosich, «Highly efficient design osf spectrally engineered whispering gallery mode microlaser resonators », Otpical and quantum Electronics, Vol. 35, pp.545-559, 2003.

[3] K.C. Chang, V. shah and T.Tamir, « Scattering and guiding of waves by dielectric gratings with arbitrary profiles », Journal of Optical Society of America, Vol. 70, pp. 804-813, july 1980. 